\title{
Child Schizophrenia: Theory and Practice
}

\author{
Petro Petlovanyi and Anatolii Tsarkov
}

\begin{abstract}
Child schizophrenia is a mental disorder that affects brain structures, emotional and cognitive functions. The disorder is characterized by psychotic symptoms: disorder of thinking, inadequate emotional reactions, disorganized behavior, deterioration of social functioning and loss of will. Schizophrenia in children is one of the most common mental illnesses affecting up to $1 \%$ of all children. For a long time, the term "child schizophrenia" was used to refer to various disorders that had similarities with each other. Children were referred to schizophrenics if they had borderline symptoms. Child schizophrenia develops gradually; sudden onset it is not common. Schizophrenic child requires help from multidisciplinary team members including child and adolescent psychiatrist and all possible levels of psychotherapy and psychological interventions. This article discusses classification, clinical presentation, all possible treatment and rehabilitation of children with schizophrenia.
\end{abstract}

Index Terms - Child and adolescent psychiatry, schizophrenia, child schizophrenia, child depression, ADHD, antipsychotics.

\section{INTRODUCTION}

According to the World Health Organization (WHO), at the beginning of the XXI century, there were more than 21 million schizophrenia patients in the world, or about $1 \%$ of the world's population [1], [2]. The prevalence of schizophrenia in children under 14 years varies from 0.1 to $1 \%$ in European countries, and increases to $4 \%$ in adolescents aged 15 years and older. In 2018, according to official statistics of the Ministry of Health of Zambia, the incidence of schizophrenia, schizoaffective and delusional disorders manifested in children under 14 years was 0.8 and the prevalence was 2.8 per 100 thousand children. In adolescents from 15 to 18 years the incidence was 9.2 and the prevalence 35.3 per 100 thousand children. The level of diagnosis of child schizophrenia in different regions of the world depends largely on cultural factors and the level of stigma attached to psychiatric care for children. Psychoses in children and adolescents have been diagnosed since the 1950s [3]. In the 1960s, childhood schizophrenia was viewed as a spectrum of heterogeneous disorders: autism, psychoses and infantile dementia [3]. In 1980, childhood schizophrenia was first included in the Diagnostic and Statistical Manual of Mental Disorders 3rd Edition (DSM-III) classification as a diagnostic category with similar schizophrenia in the adult diagnostic criteria [4]. In the 1970s and 1980s, the practice of advanced diagnosis of schizophrenia took place in European countries (Ukraine, Poland and Germany), and specific forms of childhood disorder were described. In International Classification of Diseases: 10th Edition (ICD-10), Diagnostic

Published on February 12, 2020.

P. Petlovanyi, Department of Psychiatry, School of Medicine, University of Zambia (UNZA), Lusaka, Zambia.

(e-mail: p.petlovanyi@gmail.com) and Statistical Manual of Mental Disorders 4th Edition (DSM-IV) and Diagnostic and Statistical Manual of Mental Disorders 5th Edition (DSM-V) classification systems, childhood schizophrenia is not distinguished as a separate diagnostic category, and it is suggested that adult diagnostic criteria be used to diagnose schizophrenia manifesting in childhood [5], [6], [7], [8].

\section{INTERACTION OF HEREDITARY AND ENVIRONMENTAL RISK FACTORS IN CHILDHOOD SCHIZOPHRENIA}

Modern views on the etiology of schizophrenia are based on the concept of the interaction of genetic and environmental factors. Schizophrenia is conceptualized as a disorder in which a variety of environmental risk factors affect a complex set of representative genes. Hereditary factors are decisive in the etiology of schizophrenia, but the environment in which gene polymorphism manifests also influences the course of the disease. The manifestation of schizophrenia in childhood is a consequence of impaired development of dopaminergic and glutamate neurotransmission systems and is caused by the interaction between genetic susceptibility to the disorder, the action of the factors provoking and inhibiting exacerbation of psychosis. Factors such as genetic risk and neurobiological dysfunctions of the central nervous system contribute to its manifestation. Adverse environmental factors that directly provoke exacerbation qualify as trigger factors. Protective factors include factors that reduce the likelihood of schizophrenia manifestation of individuals at risk: high levels of development of intelligence, social skills and sufficient social support for the child in the family. Despite the genetic predisposition to schizophrenia, the likelihood of becoming sick is significant only when a person with a predisposition to the disorder is exposed to adverse environmental factors and does not have sufficient resources to resist it. The question remains how genetic polymorphism correlates with the clinical diversity of individual phenotypes and through which mechanisms environmental factors determine the relevance of clinical manifestations in specific patients. Schizophrenia genotype is characterized by a certain variability and polymorphism of different in severity of new and old mutations.

A number of rare deletions and duplications of DNA sequences are associated with an increased risk of schizophrenia. The association with schizophrenia of the RELN gene polymorphism, microdeletions 22q11.2, 1q21.1, $15 \mathrm{q} 13.3$ and microduplications $16 \mathrm{p} 11.2$ has been proved [9], [10]. Recently, members of an international scientific

A. Tsarkov, Department of Psychiatry, School of Medicine, University of Zambia (UNZA), Lusaka, Zambia \& Chainama Hills College Hospital $(\mathrm{CHCH})$, Lusaka, Zambia.

(e-mail: anatoliydoc@gmail.com) 
consortium have identified a number of new mutations that are significantly associated with schizophrenia. Most of them belong to the new mutations that are observed in the child, but are absent from her parents. Such variants of gene polymorphism can be observed in more than ten chromosome loci, in particular ZNF804A at 2q32.1, NRGN at 11q24.2, TCF4 at 18q21.2, AMBRA1 at 11p11.2, VRK2 at 2p15.1, MIR137 at 1p21.3, PCGEM1 at 2q32.3, CSMD1 at 8p23.2, MMP16 at 8q21.3, CNNM2 at 10q24.3, BRP44 at 1q24.2, WHSC1L1 at 8p12, and TSPAN18 at 11p11.2 [11].

\section{ENVIRONMENTAL RISK FACTORS OF SCHIZOPHRENIA DURING PRENATAL PERIOD AND WITHIN FIRST YEAR AFTER THE BABY IS BORN}

It is believed that environmental factors interact with genetic factors during the early stages of nervous system formation and cause disturbances in neurotransmitter systems differentiation, for example, leading to excessive dopamine receptor density. Such features of the development of the dopaminergic system are predictors of human affinity for tic disorders and schizophrenia.

Numerous studies have resulted in the identification of environmental risk factors for schizophrenia that persist during the first year of life, long before any of its symptoms can be identified. These factors include obstetric complications, antenatal and postnatal infections, other factors that are active during the period of intrauterine brain development.

Children born in the winter or early spring in the Northern Hemisphere are at greater risk of schizophrenia later in life than those born at other times of the year [12]. The design effect for the seasons of birth in the Northern Hemisphere is 1.07 at a confidence interval of 1.05-1.08. This means that the specific risk of developing schizophrenia for birth in the winter and spring in the Northern Hemisphere is 3.3\%. There are many potential mechanisms associated with the impact of the season of birth on the risk of schizophrenia: higher incidence of obstetric complications, lack of solar insulation, adverse temperature effects, seasonal changes in diet and seasonal genetic influences.

The impact of infectious diseases such as influenza carried out during pregnancy is the most studied factor that increases the risk of schizophrenia [13]. A number of gestational viral agents, especially such as rubella, cytomegalovirus and herpes simplex have been shown to be associated with birth defects in the baby's brain. Gestational infections can be attributed to a number of contributing factors of schizophrenia.

Postnatal infection may also play a significant role in the etiology of schizophrenia. Pediatric neuroinfection before to the age of 14 years correlates with a moderately higher risk of schizophrenia in adulthood. A correlation between childhood meningitis under the age of four and adult psychosis has been established. Toxoplasma gondii as an intracellular parasite has been identified as another etiologic agent acting before and after the birth of a baby and increases the risk of schizophrenia manifestation.

Non-infectious environmental agents, such as malnutrition, diabetes, maternal smoking during pregnancy and rhesus conflict, increase the risk of schizophrenia manifestation in offspring. The possible significance of low vitamin D is researched [14], [15]. A study in a Finnish population demonstrated that providing babies with vitamin $\mathrm{D}$ during the first year of life had a protective effect and reducing their risk of schizophrenia. At the same time, there was no evidence of a relationship between serum levels of vitamin $\mathrm{D}$ in the blood plasma of women in childbirth and the risk of schizophrenia in their children. Disorders of homocysteine metabolism and low levels of folate in the plasma of pregnant women are also considered to be risk factors for fetal neurodevelopment and schizophrenia manifestation in offspring in adulthood

\section{EARLY CHILDHOOD RISK FACTORS FOR SCHIZOPHRENIA}

Most environmental risk factors work for a long time, during childhood and adulthood, right before the manifestation of schizophrenia. Particular importance is attached to a number of factors acting up to the age of three. They are peculiarities of raising a child, early loss of parents, having a cold and emotionally immature mother, inability to form an object of attachment, cruel attitudes or sexual abuse at a young age, primarily by persons in the primary support group [16].

The impact of parenting on the realization of the risk of schizophrenia manifestation was first proven in Israeli study [17]. Researchers have concluded that raising children in kibbutz (a collective community in Israel that was traditionally based on agriculture) increases the risk of developing mental disorders.

The risk of manifesting schizophrenia in adulthood has a correlation with the atypical interaction in the mother-infant system [18]. Mothers of children who were diagnosed with schizophrenia and schizophrenia at adulthood were more likely to have atypical interaction in the mother-child system. Similar associations have not been confirmed for mothers of children with other psychiatric disorders, such as manic, anxiety, or depressive disorders.

Adoption of children at high family risk for schizophrenia increases this risk. Children of mothers with schizophrenia were more likely to have schizophrenia, bipolar disorder and other severe mental disorders than the control group of adoptive parents. There is ample evidence that the abuse and sexual abuse of a child increases the risk of psychotic disorders in adulthood, particularly schizophrenia.

\section{ENVIRONMENTAL RISK FACTORS FOR SCHIZOPHRENIA ASSOCIATED WITH ADOLESCENCE}

Environmental risk factors can be considered not only as potential etiologic but also precipitating factors that provoke manifestation in disorder-prone individuals or modify the course, such as the progressiveness of the latent process.

It has been established that the early and prolonged use of methamphetamine may increase the risk of developing psychoses during life. It has been shown that cases of schizophrenia in family history or the presence of premorbid schizoid or schizotypal personality traits increase the risk of psychotic symptoms when using psychostimulants. The 
impact on schizophrenia of cannabinoid abuse has also been proven. It has long been known that cannabinoid intoxication can increase the severity of psychotic symptoms and accelerate the exacerbation of a psychotic episode. There are many evidences that marijuana is a trigger for schizophrenia, especially in people who are prone to the disorder. The debate raises the question of whether marijuana can cause schizophrenia in people who use it but have no previous susceptibility to the disorder.

\section{HOW IS CHILD SCHIZOPHRENIA DIAGNOSED?}

The diagnostic process for child schizophrenia has a number of interrelated goals: early diagnosis of psychotic symptoms, prediction of specific risk groups, diagnosis of mental disorders according to the diagnostic criteria of ICD10 or DSM-V, disorders of adaptive behavior and diagnosis of comorbid psychiatric disorders. Another important goal of the diagnostic process is to establish therapeutic interaction with family and child, partnerships, reach a common vision of problems, goals and formulate an appropriate plan of action.

There were attempts to diagnose child schizophrenia as a separate disorder different from schizophrenia in adults. However, it is proven today that based on the criteria used to diagnose schizophrenia in adults, it is possible to diagnose schizophrenia in children with a high degree of reliability.

Every child with suspected schizophrenia should be examined by a child psychiatrist. Psychiatric examination should include interviews with the child and both parents. It is necessary to investigate the family history and life history of the child: to determine the presence of cases of mental illness, suicide, the influence of environmental factors, the effect of which is associated with an increased risk of schizophrenia; to find out the developmental features of the child, its strengths and weaknesses, the presence of protective factors and factors that increase the risk of exacerbation of psychosis. A psychiatric examination should also include detailed medical history.

In the physical examination we should exclude general medical causes of psychotic symptoms, first of all possible organic causes of disorders, among which the conditions are associated with acute intoxication, use of psychoactive substances, infectious and neuroinfectious, tumors and epilepsy.

Laboratory testing, electroencephalography, and neuroimaging (computerized tomography (CT) scan and Magnetic resonance imaging (MRI) scan) are not relevant for the diagnosis of schizophrenia but may be used in differential diagnosis. Some laboratory investigation, such as assessment of renal or hepatic function, may be used to monitor the safety of psychotropic treatment.

Whenever possible, we have to try to make a diagnosis within one of the standardized international classifications ICD-10 or DSM-V [19]. Diagnosis of child schizophrenia is the exclusive competence of a child psychiatrist. To establish a detailed diagnosis, it is sufficient to use generally recognized diagnostic criteria. In difficult cases for differential diagnosis, in particular in the presence of comorbid psychiatric disorders, to determine the severity of the disorder, the effectiveness and safety of care, there is a need for a formalized psychiatric examination of the child using standardized screening tools. Semi-structured interviews with parents: Kiddie-Sads-Present and Lifetime Version (K-SADS-PL) and Development and Well-Being Assessment (DAWBA) may be used for the final diagnosis of schizophrenia and for differential diagnosis with other psychiatric disorders. To assess the severity of adolescent schizophrenia symptoms and clinical dynamics in the course of psychopharmacology, the Positive and Negative Syndrome Scale (PANSS) and the Clinical Global Impression-Schizophrenia (CGI-SCH) scale are recommended. The Kaufman Brief Intelligence Test, Second Edition (KBIT-2) scale may be used to assess the level of cognitive functioning. The Vineland Adaptive Behavior Scales and The Social Responsiveness Scale are recommended for assessing adaptive behavior disorders.

\section{TREATMENT OF CHILD SCHIZOPHRENIA}

The polymorphism of clinical manifestation and its biological heterogeneity require complex application of pharmacological, psychological treatments and special forms of social support. A child psychiatrist providing specialized psychiatric care to a child with schizophrenia should take into account the stage of development of disorder, the severity and mechanisms of adaptive behavior, the presence of special educational and intellectual problems and the problem associated with the context of the family and family dynamics.

Therapeutic program should always include psychoeducation of the child, parents and mobilization of family support. The specific tasks of psychological therapy are:

- formation of adherence to therapy and adherence to therapeutic regimen;

- primary support group conflict resolution, and

- improving social functioning.

In the early manifestation (up to 13 years), disorders of cognitive function, school and social maladaptation are essential. In such case, psychosocial assistance and special educational services is important. The support of the child should be aimed at maintaining its connection with the school staff and continuing to attend school. When planning a therapy strategy, it is advisable to limit, as far as possible, the child's stay in a psychiatric unit/hospital, to ensure his or her treatment mainly in an outpatient setting, combining it with various forms of inclusive education.

Schizophrenia manifesting in puberty can adversely affect professional formation, social functioning, reproductive health, and family relationships. Such patients may benefit from special support in these areas.

\section{PSYCHOPHARMACOLOGICAL TREATMENT}

The effects of schizophrenia are considered more serious than the side effects of its treatment. Antipsychotic therapy (AT) has no alternative in the treatment of schizophrenia. Its effectiveness has been proven in numerous placebocontrolled trials. AT can significantly improve the condition of patients. Early diagnosis and initiation of treatment 
significantly improve the prognosis of the disorder. The neurobiological basis of the clinical efficacy of neuroleptics in patients with schizophrenia is a decrease in the density of dopamine receptors in the mesolimbic structures of the patient's brain, resulting in the elimination of delusions, hallucinations, and aggressive behavior.

Atypical antipsychotics also improve dopamine neurotransmission in the frontal brain, which is associated with the reduction of negative symptoms of schizophrenia. The drugs in this group have no alternative in the treatment of childhood schizophrenia. A number of controlled studies have shown that second-generation antipsychotics are no less effective in treating schizophrenia in children than conventional antipsychotic, however they have a lower risk of side effects, including effects on cognitive function [20], [21], [22].

Over the last several years, the Food and Drug Administration (FDA) has significantly expanded the list of atypical antipsychotics recommended for use in children and approved a number of drugs for use at the age of five [23], [24], [25]. Risperidone is recommended for the treatment of aggression in the autism spectrum disorder from 6 years, schizophrenia in adolescents from 13 years, as well as for short-term treatment of manic or mixed episodes of bipolar disorder in adolescents from 10 years; aripiprazole was added with identical indications for use. Subsequently, quetiapine, olanzapine, ziprasidone for the treatment of schizophrenia from 13 years of age and manic and mixed episodes of bipolar disorder in adolescents from 10 years. In Zambia, the only atypical antipsychotic recommended for use in pediatric practice is risperidone. Indications for its use are similar to those registered in the USA and Europe.

\section{USE OF ATYPICAL ANTIPSYCHOTICS IN PEDIATRIC PRACTICE}

In order to ensure the effectiveness and safety of the AT in pediatric psychiatric practice, the requirements for the quality of diagnosis should be raised first. It is necessary to make sure that the child is diagnosed in strict accordance with the diagnostic criteria of ICD-10 and/or DSM-V.

In the absence of a therapeutic response to AT, it is first and foremost necessary to ensure that the child and the family correctly understand and steadily follow the dosage regimen. Parents can change the frequency of dosing, reduce doses, disrupt the dosing regimen, and forget to give medication to the child in due time without consulting the doctor. Sometimes, when giving a child a drug, parents allow her to leave without being convinced that she has swallowed them. The child can hide the pill under the tongue or cheek and then spit it out. In such cases, oral dispersion tablets and drinking solutions can be very useful. It is necessary to first establish the relevance of the actual administration of the medication, and then evaluate the effectiveness of treatment. The use of AT in child schizophrenia should be long-lasting and continuous.

Combination therapy (administration of two antipsychotics or more) should be avoided. Properly selected treatment allows not to prescribe additional drugs. Doses of antipsychotic for children should be selected individually, by gradual increasing to the minimum effective dose. Drug withdrawal should also be performed very slowly. In children, it is recommended that the frequency of taking antipsychotic more often than in adults: from 6 to 10 years, the daily dose is recommended to be divided into three doses; from 11 to 14 years - for two doses. This dosage regimen reduces the peak concentrations and associated side effects and also maintains a stable serum concentration in young children who have a faster liver metabolism. The effectiveness of AT in children is largely related to adherence to regimens and healthy lifestyle.

AT may lead to increased appetite and metabolic disorders. It is necessary to control the amount of food consumed by the child and its calorie content. By following dietary guidelines and sufficient physical activity, the risk of developing metabolic syndrome can be significantly reduced. Parents should be trained to monitor side effects and take measures to improve tolerability of treatment.

\section{GENERAL PRINCIPLES OF NON-BIOLOGICAL TREATMENT OF CHILD SCHIZOPHRENIA}

In the treatment of schizophrenia in children and adolescents, methods of psychological treatment and various forms of social support for the child and his family are important. The psychosocial interventions recommended for the rehabilitation of children and adolescents with schizophrenia include the following:

- social support for the case;

- psychoeducation of the child and his/her family members;

- social skills training;

- compliance therapy and psychoeducation (training on adherence to therapeutic regimen);

- establishing a supportive relationship.

Special psychological treatments can be used to improve social functioning, adhere to therapeutic regimen, and control of residual symptoms.

At the stage of manifest of psychotic disorders, psychoeducation with child and his/her primary support group should be aimed at overcoming existential fear and confusion, recognizing the symptoms of the disorder, its connection with biological dysfunctions of the brain activity, recognizing the need for treatment, in particular taking AT. Social support at this stage of the disorder aims to support outpatient treatment, creating conditions to avoid hospitalization.

At the stage of gradual reduction of psychotic disorders, psychoeducational work should be aimed at achieving the understanding of the positive dynamics of the symptoms of schizophrenia with AT, formation of adherence to treatment, and setting up for social function. Social support at this stage of the disorders aims at reducing the length of stay of the child in an inpatient psychiatric facility and ensuring that the child or adolescent is discharged from the psychiatric hospital as soon as possible.

At the stage of remission (after the discharge), psychoeducation and special psychological methods of treatment should be aimed at accepting the child and his/her parents of the disorder without blaming himself/herself and others, restoration of low self-esteem, lost sense of life and recognition of the need for long-term maintenance therapy. In complex remission therapy adolescent schizophrenia, we 
recommend the use of short-term psychotherapy. Short-term psychotherapy can be aimed at rebuilding a teenager after a psychotic episode. This form of psychological treatment consists in the formation of skills to identify painful changes, to create the conditions for adaptation to the changes caused by psychotic disorders and the skills to control the relapse of schizophrenic symptoms.

\section{CONCLUSION}

Psychiatric care for children and adolescents with schizophrenia should be provided at destigmatized and acceptable outpatient setting. The stay of the child in inpatient psychiatric wards should be short-term and wellmotivated. An early intervention program for schizophrenia, manifesting between the ages of 10 and 18, should include: engaging educators for early rehabilitation, psychosocial work and family therapy using stress management and social skills training. Early psychosocial assistance in combination with long-term treatment with atypical antipsychotic is more effective in the correction of cognitive impairment and behavioral disorders than monotherapy with antipsychotic. When using atypical antipsychotic therapy in combination with psychosocial interventions, the quality of life and social functioning of children with schizophrenia are improved. Adherence to therapy is a prerequisite for its effectiveness. The child and his/her parents should be direct participants in the therapeutic process, be involved in monitoring side effects, taking measures to minimize the side effects of treatment and improving its tolerability.

\section{ACKNOWLEDGMENT}

Authors wish to acknowledge continuous help and support of Professor Trevor Kaile (Dean, School of Medicine, University of Zambia - UNZA), Doctor Elliot Kafumukache (Acting Dean, School of Medicine, University of Zambia UNZA), and Doctor Ravi Paul (Head of Department, Department of Psychiatry, University of Zambia - UNZA), who made writing this paper possible.

\section{REFERENCES}

[1] World Health Organization. (2018). Schizophrenia. Available at: https://www.who.int/news-room/fact-sheets/detail/schizophrenia (Accessed on 1st October, 2019).

[2] World Federation for Mental Health. (2014). Living a healthy life with schizophrenia: paving the road to recovery. Section V: Time to act. Available at: https://www.who.int/mental health/world-mental-healthday/paper wfmh.pdf (Accessed on 1st October, 2019).

[3] Remschmidt, H. E., Schulz, E., Martin, M., Warnke, A. \& Trott, G. E. (1994). Childhood-Onset Schizophrenia: History of the Concept and Recent Studies. Schizophrenia Bulletin, 20(4): 727-745.

[4] American Psychiatric Association. (1980). DSM-III. Diagnostic and Statistical Manual of Mental Disorders (Third Edition): 2nd Printing edition.

[5] American Psychiatric Association. (1994). DSM-IV: Diagnostic and Statistical Manual of Mental Disorders: 4th Edition.

[6] American Psychiatric Association. (2013). Diagnostic and Statistical Manual of Mental Disorders, Fifth Edition (DSM-5(TM)): 5th Edition.

[7] World Health Organization. (1992). The ICD-10 Classification of Mental and Behavioural Disorders: Clinical Descriptions and Diagnostic Guidelines: 1st Edition.
[8] Phiri, C., Tsarkov, A., Petlovanyi, P., \& Lingenda, G. Factors Contributing To Oral Diseases and Treatment Needs amongst Mental Patients at Chainama Hills College Hospital, Lusaka, Zambia. Available

https://pdfs.semanticscholar.org/eb6c/a411d94f5adeb7f68fdaa3c728a ad2038f85.pdf (Accessed on 10th October, 2019).

[9] D. Yu. Galaktionova, D. Y., Gareeva, A. E., Khusnutdinova, E. K. \& Nasedkina, T, V. (2014). Association of SLC18A1, TPH1, and RELN gene polymorphisms with risk of paranoid schizophrenia. Molecular Biology, 48(4): 546-555.

[10] Haldeman-Englert, C. R. \& Jewett, T. (2015). 1q21.1 Recurrent Microdeletion. GeneReviews ${ }^{\circledR}$ (Internet). Seattle (WA): University of Washington, Seattle; 1993-2019. Available at: https://www.ncbi.nlm.nih.gov/books/NBK52787/ (Accessed on 4th October, 2019).

[11] Steinberg, S., de Jong, S., Irish Schizophrenia Genomics Consortium, Andreassen, O. A., Werge, T., Børglum, A. D. \& Stefansson, K. (2011). Common variants at VRK2 and TCF4 conferring risk of schizophrenia. Human molecular genetics, 20(20), 4076-4081. doi:10.1093/hmg/ddr325.

[12] McTigue, O., \& O'Callaghan, E. (2000). Season of Birth as a Risk Factor. International Journal of Mental Health, 29(3), 66-78.

[13] Boksa P. (2008). Maternal infection during pregnancy and schizophrenia. Journal of psychiatry \& neuroscience: JPN, 33(3), 183185.

[14] Yüksel, R. N., Altunsoy, N., Tikir, B., Cingi Külük, M., Unal, K., Goka, S., ... Goka, E. (2014). Correlation between total vitamin D levels and psychotic psychopathology in patients with schizophrenia: therapeutic implications for add-on vitamin D augmentation. Therapeutic advances in psychopharmacology, 4(6), 268-275.

[15] McGrath, J., Brown, A., \& St Clair, D. (2011). Prevention and schizophrenia--the role of dietary factors. Schizophrenia bulletin, 37(2), 272-283.

[16] Tsarkov, A., \& Petlovanyi, P. (2017). Omega-3 Fatty Acids as an Alternative Treatment for Children with Attention Deficit Hyperactivity Disorder. Imperial Journal of Interdisciplinary Research (IJIR), 3, 1378-1380. Available at: https://pdfs.semanticscholar.org/42bd/c75304c050d502670c7e8c86fd df5f5079ce.pdf (Accessed on 12th October, 2019).

[17] Nagler, S. \& Mirsky, A. F. (1985). Introduction: The Israeli High-Risk Study. Schizophr Bull., 11(1): 19-29.

[18] Davidsen, K. A., Harder, S., MacBeth, A., Lundy, J. M., \& Gumley, A. (2015). Mother-infant interaction in schizophrenia: transmitting risk or resilience? A systematic review of the literature. Social psychiatry and psychiatric epidemiology, 50(12): 1785-1798.

[19] Tsarkov, A., \& Petlovanyi, P. (2019). Neuropsychiatric Aspects of a Common Problem: Stroke. European Journal of Medical and Health Sciences, 1(3). https://doi.org/10.24018/ejmed.2019.1.3.64 Available at:

https://pdfs.semanticscholar.org/e830/852f04b7b788300b6f23f295e1 0327c858e5.pdf (Accessed on 12th October, 2019).

[20] Li M. (2016). Antipsychotic-induced sensitization and tolerance: Behavioral characteristics, developmental impacts, and neurobiological mechanisms. Journal of psychopharmacology, 30(8): 749-770.

[21] Tsarkov, A., \& Petlovanyi, P. The Role of Lamotrigine in the Treatment of Bipolar Depression. Available at: https://pdfs.semanticscholar.org/bd92/bbbe8a986a155e0684fd6f2756 9ebd895d6e.pdf (Accessed on 10th October, 2019).

[22] Reyes, M., Croonenberghs, J., Augustyns, I. \& Eerdekens, M. (2006). Long-term use of risperidone in children with disruptive behavior disorders and subaverage intelligence: efficacy, safety, and tolerability. J. Child Adolesc. Psychopharmacol., 16(3): 260-272.

[23] Food and Drug Administration. (2015). Atypical Antipsychotic Medications: Use in Pediatric Patients. Available at: https://www.cms.gov/Medicare-Medicaid-Coordination/FraudPrevention/Medicaid-Integrity-Education/Pharmacy-Education$\underline{\text { Materials/Downloads/atyp-antipsych-pediatric-factsheet11-14.pdf }}$ (Accessed on 9th October, 2019).

[24] Tsarkov, A., \& Petlovanyi, P. (2016). Bipolar Disorder in Child Psychiatric Practice: A Case Report. Medical Journal of Zambia, 43(1), 41-46. Available

at: https://www.ajol.info/index.php/mjz/article/viewFile/142808/132555 (Accessed on 9th October, 2019).

[25] Tsarkov, A., \& Petlovanyi, P. Depressive Disorder in Child Psychiatric Practice: A. The Health Press, 9. Available at: http://znphi.co.zm/thehealthpress/wpcontent/uploads/2017/09/THPZ V1 I5 May final.3.pdf (Accessed on 10th October, 2019). 